\title{
Evidence for in vivo transport of bioactive nitric oxide in human plasma
}

\author{
Tienush Rassaf, ${ }^{1,2}$ Michael Preik, ${ }^{1}$ Petra Kleinbongard, ${ }^{1}$ Thomas Lauer, ${ }^{1}$ Christian Heiß, ${ }^{1}$ \\ Bodo-Eckehard Strauer, ${ }^{1}$ Martin Feelisch, ${ }^{2}$ and Malte Kelm ${ }^{1}$
}

\author{
${ }^{1}$ Department of Medicine, Division of Cardiology, Pulmonary Diseases and Angiology, Heinrich-Heine-Universität, \\ Düsseldorf, Germany \\ ${ }^{2}$ Department of Molecular and Cellular Physiology, Louisiana State University Health Sciences Center, \\ Shreveport, Louisiana, USA
}

Address correspondence to: Malte Kelm, Heinrich-Heine-Universität Düsseldorf Medizinische Klinik und Poliklinik B, Klinik f. Kardiologie, Pneumologie, Angiologie, Moorenstraße 5, D-40225 Düsseldorf, Germany.

Phone: 49-211-8118800; Fax: 49-211-8117851; E-mail: kelm@med.uni-duesseldorf.de.

Tienush Rassaf and Michael Preik contributed equally to this work.

Received for publication January 7, 2002, and accepted in revised form March 19, 2002.

\begin{abstract}
Although hitherto considered as a strictly locally acting vasodilator, results from recent clinical studies with inhaled nitric oxide (NO) indicate that $\mathrm{NO}$ can exert effects beyond the pulmonary circulation. We therefore sought to investigate potential remote vascular effects of intra-arterially applied aqueous NO solution and to identify the mechanisms involved. On bolus application of NO into the brachial artery of 32 healthy volunteers, both diameter of the downstream radial artery and forearm blood flow increased in a dose-dependent manner. Maximum dilator responses were comparable to those after stimulation of endogenous NO formation with acetylcholine and bradykinin. Response kinetics and pattern of NO decomposition suggested that despite the presence of hemoglobin-containing erythrocytes, a significant portion of $\mathrm{NO}$ was transported in its unbound form. Infusion of $\mathrm{NO}(36 \mu \mathrm{mol} / \mathrm{min})$ into the brachial artery increased levels of plasma nitroso species, nitrite, and nitrate in the draining antecubital vein (by $<2$-fold, 30 -fold, and 4-fold, respectively), indicative of oxidative and nitrosative chemistry. Infused $\mathrm{N}$-oxides were inactive as vasodilators whereas $S$-nitrosoglutathione dilated conduit and resistance arteries. Our results suggest that NO can be transported in bioactive form for significant distances along the vascular bed. Both free NO and plasma nitroso species contribute to the dilation of the downstream vasculature.
\end{abstract}

J. Clin. Invest. 109:1241-1248 (2002). DOI:10.1172/JCI200214995.

\section{Introduction}

Previous experimental studies have demonstrated that the endothelium plays a crucial role in the maintenance of vascular homeostasis, including the control of thrombosis, the interaction of platelets and leukocytes with the vessel wall, and regulation of vascular tone and growth (1-3). Among the various mediators released by the endothelium, nitric oxide (NO) is of major importance. Due to technical difficulties in the preparation and handling of authentic $\mathrm{NO}$, analytical problems with its detection in biological media, and a rather complex metabolism in blood, relatively little is known about the transport and biological activity of NO in the human circulation. Recent estimates of the half-life of NO in blood range from 0.05 to 1.8 milliseconds (4-6). However, experimental evidence obtained in laboratory rats suggests that once NO enters the circulation it is not merely inactivated but is in part transported to and interchanges with other compartments (7). These considerations raise the fundamental question as to whether or not authentic NO may exert biological effects distal to its site of applica- tion. Various metabolic routes may contribute to the breakdown and/or conversion of NO in the vascular system, and these may differ between cellular compartments and with changes in the relative concentration of different blood constituents. Matters are further complicated by the fact that, due to a variable plasma/blood cell ratio, the metabolic routes for $\mathrm{NO}$ are likely to vary along the vascular tree. The two probably most important compartments in this context are plasma and red blood cells (RBCs) (8).

Blood constitutes approximately $60 \%$ of plasma. The latter thus represents a potentially significant compartment for NO metabolism (9). The major immediate breakdown product of $\mathrm{NO}$ in human plasma is nitrite $\left(\mathrm{NO}_{2}^{-}\right)$, which at physiological concentrations is devoid of vasodilator activity (10). These findings, however, do not exclude that under certain conditions nitrite may further react with plasma or cellular constituents. Studies in experimental animals revealed that redox-active thiols, which are abundantly present in plasma (11), can incorporate NO and transport it throughout the mammalian circulation 
in the form of bioactive $S$-nitrosothiols (RSNOs) (12). In the presence of oxygen, $S$-nitrosoalbumin (SNOAlb) is thought to represent the major reaction product of NO with plasma (13-16). Although there is no doubt as to its existence in vivo, mechanisms of formation and subsequent release of NO from SNOAlb and other RSNOs are poorly understood (17, 18 ), and no data are available on any of these processes in the human circulation.

The other major compartment for NO metabolism in blood is represented by the RBCs, for which three principal routes of $\mathrm{NO}$ interactions are envisioned. In aqueous solution, $\mathrm{NO}$ reacts rapidly with oxyhemoglobin (oxyHb) to form nitrate $\left(\mathrm{NO}_{3}^{-}\right)$and methemoglobin (19). Although this reaction has received widespread recognition as the major inactivation pathway of $\mathrm{NO}$ in vivo, recent results obtained in humans suggest that this assumption may not be valid under all conditions (10). Alternatively, NO may bind to the heme group of deoxyhemoglobin (deoxyHb) to form nitrosylhemoglobin $(\mathrm{NOHb})(8)$. The latter has been detected in the blood of patients receiving nitroglycerin or inhaling $\mathrm{NO}$ gas (20-22). A third possibility is the reaction of $\mathrm{NO}$, or a higher oxidation product such as $\mathrm{NO}_{2}$ or $\mathrm{N}_{2} \mathrm{O}_{3}$, with cysteine-93 of the $\beta$-globin chains $\left(\beta-\mathrm{Cys}{ }^{93}\right)$ of hemoglobin, leading to formation of an $S$-nitrosated derivative of oxyhemoglobin ( $\mathrm{SNOHb}$ ), which has been suggested to participate in the regulation of blood flow (23) and platelet aggregability (24). Whereas the reactions of $\mathrm{NO}$ with oxyHb and deoxyHb are well characterized $(8,9)$, the potential role of $\beta$-Cys ${ }^{93}$ nitrosation by NO has so far been established in animal models only and challenged in humans (20).

In view of the lack of information on the action of authentic NO in humans, we sought to explore the possibility that aqueous NO solution exerts regional vasodilator effects on conduit and resistance arteries of the human forearm circulation, and that this may, at least in part, be mediated by the formation of plasma RSNOs. To the best of our knowledge, no data have been reported on the vasorelaxant effects of NO in the peripheral vasculature in humans, presumably because of the technical obstacles associated with the production of sterile aqueous NO solutions as well as the supposed ultra-short half-life and rapid clearance of NO from blood. The results of our present study challenge this current dogma. Here we demonstrate, for the first time to our knowledge, that aqueous solutions of authentic NO can be safely applied into the brachial artery and that $\mathrm{NO}$ consecutively dilates downstream conduit and resistance arteries. The rapid formation of substantial amounts of nitrite, along with the rapid onset of vasodilation mimicking flow responses after stimulation of endogenous NO synthesis, suggests that NO is transported as such at significant distances in human plasma along the vascular tree. We furthermore show that part of this effect is mediated via in vivo formation of plasma RSNOs, whereas the major NO metabolites, nitrite and nitrate, are inactive as vasodilators.

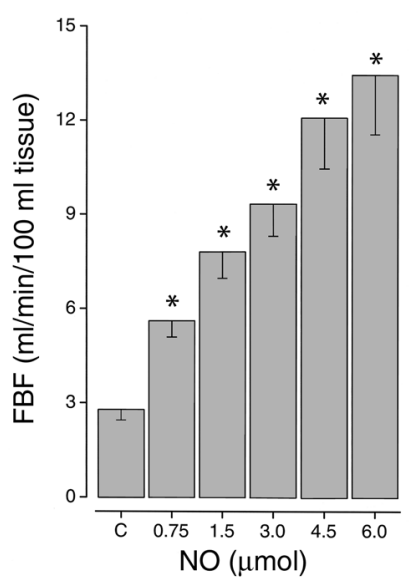

Figure 1

Dose-response curve for changes in FBF following application of increasing doses of NO applied into the brachial artery $(n=15)$. * Significant differences from base line. C, control.

\section{Methods}

Chemicals. Argon (99.998\%; N5.0) and NO (99.5\%; N3.0) were purchased from Linde AG (Düsseldorf, Germany). $S$-nitrosoglutathione (GSNO) was from Alexis Deutschland GmbH (Grünberg, Germany), and sodium nitroprusside was from Schwarz Pharma AG (Monheim, Germany). All other chemicals were of analytical grade and obtained from Merck Eurolab $\mathrm{GmbH}$ (Darmstadt, Germany). GSNO solutions were prepared fresh in saline and kept in the dark until use.

Preparation of saline NO stock solutions. Saline stock solutions of authentic NO were prepared under an inert atmosphere in a custom-designed all-glass setup. Before introduction of $\mathrm{NO}$, oxygen was removed by flushing the entire system, comprising two washing bottles and a three-neck flask connected in series, for 80 minutes with argon. Traces of oxygen in the argon source were scrubbed by passing the gas through a solution of $5 \%$ pyrogallol in saturated aqueous $\mathrm{KOH}$. Then, the saline $(0.9 \% \mathrm{NaCl})$ solution contained in the three-neck flask was bubbled for 45 minutes with NO gas, which has been freed from higher $\mathrm{N}$-oxides by passing it through a $20 \% \mathrm{NaOH}$ solution. The resulting NO-saturated solution was subsequently filtered through a $0.2-\mu \mathrm{m}$ filter (Millipore GmbH, Eschborn, Germany) under a laminar air flow cabinet and kept at $21 \pm 1^{\circ} \mathrm{C}$ in septum-sealed glass containers. The NO concentration of these solutions was typically $1.5 \pm 0.2$ $\mathrm{mM}$, as determined using the oxyHb technique, and its stability was checked at regular intervals. These solutions were used within 1-3 hours after preparation, during which time the concentration did not change by more than $2 \%(n=8)$.

Vascular studies. Healthy volunteers were screened by clinical history, physical examination, and routine chemical analysis. None of the subjects was on a regular medication, was a smoker, or revealed present or past evidence of cardiovascular diseases known to affect endothelial function, such as hypertension, hypercholesterolemia, chronic heart failure, and diabetes mellitus. Further laboratory tests also excluded elevated levels of C-reactive protein, confirming the absence of inflammatory processes. Participants in the 


\section{Figure 2}

Comparison of the maximal dilator effects of endogenous and exogenous $\mathrm{NO}$ in the human forearm. The endotheliumdependent dilators $\mathrm{ACH}$ and $\mathrm{BK}$, the $\mathrm{NO}$ donor $\mathrm{SNP}$, and authentic NO (NO) were applied in random order at maximally effective doses $(n=8)$. * Significant differences from base line. C, control.

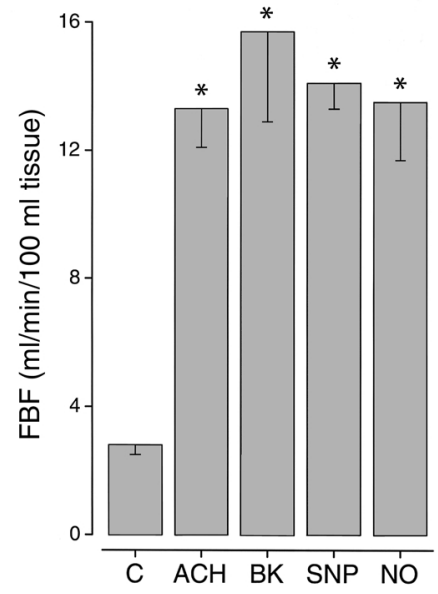

study abstained from drinking caffeine-containing beverages at least 12 hours prior to investigation and were studied in supine position in a quiet, air-conditioned room $\left(21^{\circ} \mathrm{C}\right)$ between 8 and $11 \mathrm{am}$. The study protocol was approved by the ethics committee of the HeinrichHeine-University, and all subjects gave written informed consent before participating in the study.

Forearm blood flow (FBF) was measured simultaneously in both arms using standard techniques of mercury-in-rubber strain-gauge plethysmography and expressed as $\mathrm{ml} / \mathrm{min} / 100 \mathrm{ml}$ tissue (25). For each determination, five FBF measurements were performed and the results averaged. A $2 \mathrm{~F}$ catheter was inserted into the brachial artery of the nondominant arm for local drug infusion and continuous measurement of intra-arterial blood pressure. A $4 \mathrm{~F}$ catheter was placed into the antecubital vein for blood sampling. The radial artery was scanned with a $15-\mathrm{MHz}$ linear array transducer (SONOS 5500; Agilent Technologies Deutschland $\mathrm{GmbH}$, Böblingen, Germany) in a longitudinal section proximal to the wrist. An arterial segment was considered suitable for investigation when, after optimization of depth and gain settings, an anatomical marker and clear images of both the anterior and posterior wall could be assessed. The position of the transducer was then marked on the skin, and the arm remained in this position throughout the study. Resting scans were recorded after each intervention. All images were documented on magneto-optical disks, transferred to a personal computer, and then converted into standard TIFF format. After calibration, arterial diameters were measured at end-diastole by an automated analysis system, as previously described (26).

Blood sampling. Arterial and venous blood was diluted $1: 5$ in $0.9 \% \mathrm{NaCl}$ supplemented with $\mathrm{N}$-ethylmaleimide (final concentration $5 \mathrm{mM}$ ), heparin (10 $\mathrm{IU} / \mathrm{ml})$, and EDTA $(2 \mathrm{mM})$ and centrifuged for 20 minutes at $800 \mathrm{~g}$ and $4^{\circ} \mathrm{C}$ immediately after sample drawing. The separated plasma samples were stored on ice. Following each application of NO, blood samples were checked photometrically for methemoglobin formation (27).
Determination of plasma RSNO, nitrite, and nitrate. Plasma concentrations of RSNOs were determined by a gas phase chemiluminescence-based technique following reductive cleavage to $\mathrm{NO}$, essentially as previously described (15). Although no attempts were made in the present study to positively identify the product of reaction of NO with plasma constituents, earlier studies in human plasma have shown that the majority of the NO bound resides at the $\mathrm{SH}$ group of serum albumin (14, $15)$. Nitrite was removed by reaction with $0.5 \%$ sulfanilamide in $0.1 \mathrm{M}$ hydrochloric acid to form a stable diazonium salt that is not converted to NO under the applied conditions (15). Nitrate concentrations in plasma were determined by HPLC with direct ultraviolet detection, essentially as previously described (28).

Study protocols. A preliminary set of investigations was performed in order to assess (a) the appropriate mode of application, comparing bolus injections with short-term infusions $(0.1-24 \mathrm{ml} / \mathrm{min}$ ) using $\mathrm{NO}$ stock solutions of $1.5 \mathrm{mmol} / \mathrm{l}$; and (b) suitable dose ranges with regard to the threshold (starting at $0.0075 \mu \mathrm{mol}$ ) and maximum dose of $\mathrm{NO}(6 \mu \mathrm{mol})$, and the kinetics $(n=10)$. Thereafter, 15 subjects received local intra-arterial bolus injections of $\mathrm{NO}$ at increasing doses of $0.75-6.0 \mu \mathrm{mol}$ (Figure 1). Maximal increases in FBF and the kinetics of the biological response to $\mathrm{NO}$ were determined. To compare the dilator efficacy of saline $\mathrm{NO}$ solutions with that of

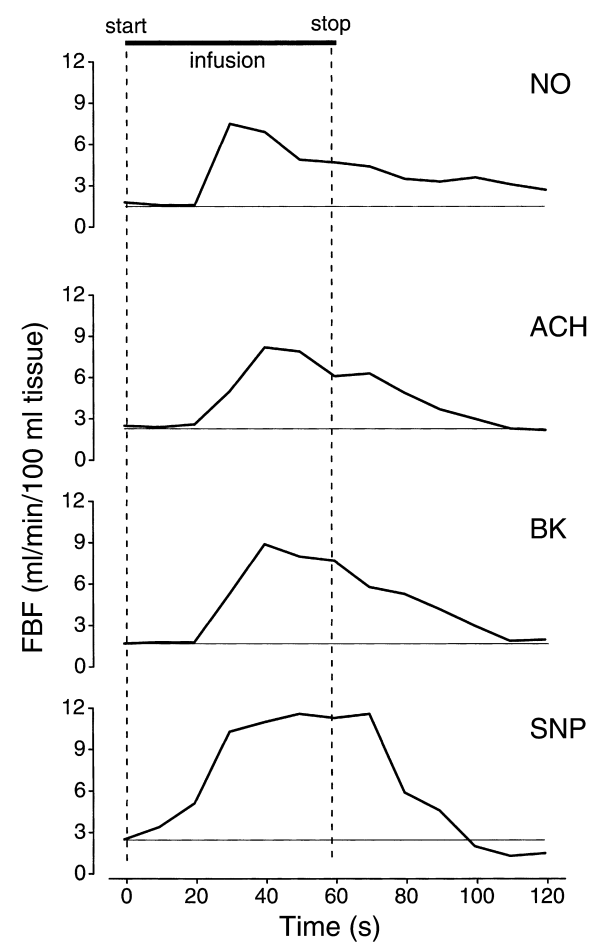

Figure 3

Comparison of the dilator kinetics of endogenous and exogenous $\mathrm{NO}$ in human forearm resistance vessels. Authentic NO (NO), the endothelium-dependent dilators $\mathrm{ACH}$ and $\mathrm{BK}$, and the $\mathrm{NO}$ donor SNP were infused for 1 minute at maximally effective doses. Each line represents the average time course of changes of FBF of three individuals. Note the sustained flow response to authentic NO. 
a

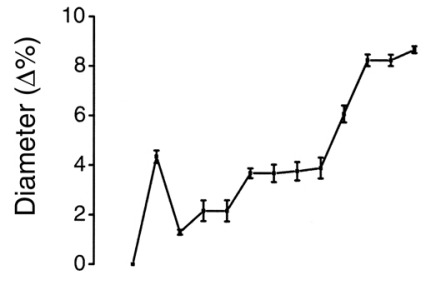

b

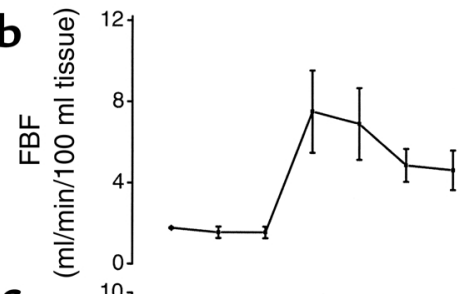

C

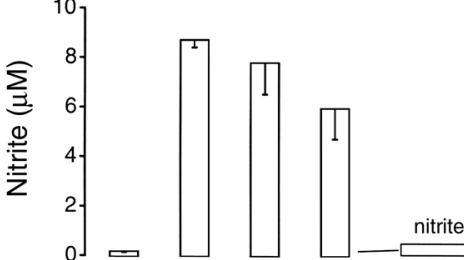

d

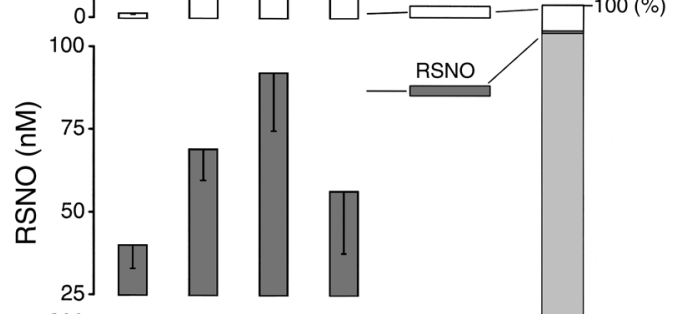

e

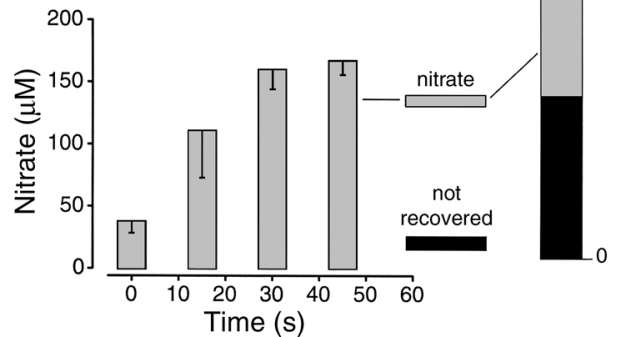

Figure 4

Dilator response to aqueous NO in conduit (a) and resistance (b) arteries as compared with the formation of the NO-related metabolites nitrite (c), RSNO (d), and nitrate (e). Radial artery diameter and FBF during 1 -minute infusion of $36 \mu \mathrm{mol} N \mathrm{NO}$ into the brachial artery were measured by high-resolution ultrasound and plethysmography $(n=3)$. In parallel, plasma concentration of the respective NO metabolites was determined from blood drawn from the antecubital vein. The bar chart (f) indicates the relative proportion (0 to $100 \%$ ) of recovered $\mathrm{N}$-oxides (nitrite, nitrate, and RSNO) formed after application of $36 \mu \mathrm{mol} \mathrm{NO}$, assuming that steady-state conditions were reached after 30 seconds.

endogenous $\mathrm{NO}$ and exogenous NO donors, the endothelium-dependent vasodilators acetylcholine $(\mathrm{ACH}, 60 \mathrm{nmol} / \mathrm{min})$ and bradykinin $(\mathrm{BK}, 200$ $\mathrm{nmol} / \mathrm{min}$ ) and the NO donor sodium nitroprusside (SNP, $34 \mathrm{nmol} / \mathrm{min}$ ) were infused into the brachial artery in random order at their maximally effective doses $(n=8)$ determined in previous studies in our laboratory (25) (Figure 2). Furthermore, the onset and offset of changes in FBF were compared after 1 minute of intraarterial infusion of $\mathrm{NO}, \mathrm{ACH}, \mathrm{BK}$, and SNP (Figure 3).
In another set of investigations, three subjects received, for 1 minute, an intra-arterial infusion of 36 $\mu \mathrm{mol} N \mathrm{NO}$ into the brachial artery; changes in diameter of the downstream radial artery and forearm resistance vessels were measured by high-resolution ultrasound and venous occlusion plethysmography (Figure 4). In parallel, blood samples were drawn from the draining antecubital vein for determination of nitrite, nitrate, and RSNOs (Figure 4). Injections or infusions of matched volumes of $0.9 \% \mathrm{NaCl}$ served as controls. To estimate the relative proportion of metabolites that originated from infused NO, we calculated the amounts of recovered species (Figure 4f) from the measured concentrations corrected for baseline levels and the respective flow increases, which were at almost steady-state conditions between 30 and 45 seconds (Figure $4 \mathrm{~b}$ ).

To identify which of the detected plasma N-oxides accounts for the observed vasodilation upon infusion of saline NO solution, we administered exogenous nitrite and nitrate (each $36 \mu \mathrm{mol} / \mathrm{min}$ ) and the low-molecular weight nitrosothiol GSNO (5 $\mathrm{nmol} / \mathrm{min}$ ) into the brachial artery (each for 5 minutes) and measured changes in diameter of radial artery and FBF (Figure $5 ; n=3$ ).

Statistical analysis. Data are reported as the mean \pm SEM, and two-sided $P$ values of less than 0.05 were considered significant. ANOVA for repeated measures followed by a Newman-Keul post-hoc test was used to test for differences in FBF between control and drug infusion. Data processing was performed using the software package SPSS (release 8.0; SPSS Science, Chicago, Illinois, USA).

\section{Results}

The study population comprised 32 healthy individuals, with a mean age of $31 \pm 4$ years, a mean body weight of $71 \pm 8 \mathrm{~kg}$, and a mean body height of $177 \pm 9 \mathrm{~cm}$. Mean arterial blood pressure $(81 \pm 11 \mathrm{mmHg})$, heart rate (67 \pm 7 beats per minute), and FBF in the contralateral arm were not significantly affected by infusion of either vasoactive substance. No bleeding complications, allergic reactions, pain sensations, edema, or any other unwanted side effects were observed either during or after application of aqueous NO solution, including in a 3-month follow-up.

Vasodilator effects of authentic NO. Unexpectedly, the dilator action of NO lasted considerably longer than predicted from its estimated half-life in blood. Irrespective of the amount of NO applied, forearm resistance vessel dilation returned to $50 \%$ of its maximal response after $28 \pm 4$ seconds. NO increased FBF in a dose-dependent manner from $2.8 \pm 0.3$ to maximally $13.5 \pm 1.8$ $\mathrm{ml} / \mathrm{min} / 100 \mathrm{ml}$ tissue $(n=15$; Figure 1$)$. The maximal increase in FBF following application of aqueous NO solution was comparable to that observed with endothelium-derived NO following infusion of maximally effective doses of $\mathrm{ACH}, \mathrm{BK}$, or exogenous $\mathrm{NO}$ released from $\operatorname{SNP}(13.5 \pm 1.8,13.3 \pm 1.2,15.7 \pm 2.8$, and $14.1 \pm 0.8 \mathrm{ml} / \mathrm{min} / 100 \mathrm{ml}$ tissue) (Figure $2 ; n=8$ ). The 
onset of changes in FBF was almost identical after infusion of $\mathrm{NO}, \mathrm{ACH}, \mathrm{BK}$, or SNP, suggesting that the initial part of the overall flow response elicited by each of the tested vasodilators was mediated by NO itself. Most strikingly, the dilatory responses to NO lasted longer than those elicited by stimulation of endogenous NO synthesis or application of SNP, returning to base line only after the second to third minute. A comparison of the dilator response to $\mathrm{NO}(36 \mu \mathrm{mol} \mathrm{NO}$ for 1 minute) in conduit and resistance arteries of the forearm circulation revealed that downstream radial artery dilation preceded that of resistance vessels (Figure 4, a and b). In vivo formation of S-nitrosothiols, nitrite, and nitrate. Basal concentrations of NO-related metabolites determined in the venous plasma of three individuals amounted to $40 \pm 7 \mathrm{nmol} / 1 \mathrm{RSNOs}, 172 \pm 35 \mathrm{nmol} / 1$ nitrite, and $38 \pm 9 \mu \mathrm{mol} / 1$ nitrate. A 1 -minute infusion of $36 \mu \mathrm{mol} \mathrm{NO}$ into the brachial artery increased basal levels of plasma RSNOs in the draining antecubital vein by $175 \%$. In parallel, venous plasma levels of nitrite and nitrate increased by more than a factor of 30 and 4 , respectively $(n=3$; Figure 4$)$. A mass analysis of the recovered NO metabolites after passage through the forearm microvasculature revealed that more than $70 \%$ resided in the plasma fraction (Figure 4, c-e), with the following rank order: nitrate > nitrite $>$ RSNOs.

Vasodilator effects of S-nitrosotbiols, nitrite, and nitrate. To compare the dilator effect of intra-arterially applied aqueous NO solution with that of a biologically relevant $S$-nitrosothiol as well as stable NO decomposition products, the pattern of relaxation in the downstream radial artery and resistance vessels produced by $\mathrm{NO}$ was compared to that of GSNO, nitrite, and nitrate. GSNO was found to dilate forearm conduit and resistance arteries. However, the onset of changes in FBF upon infusion of GSNO was observed at a considerably later time than that upon infusion of $\mathrm{NO}$ (compare Figures 4 and 5). In contrast, the application of exogenous nitrite and nitrate at doses equimolar to those of $\mathrm{NO}$ did not exert a dilation at all, ruling out a contribution of these metabolites to the vascular response produced by aqueous $\mathrm{NO}$ solutions.

\section{Discussion}

In the present study we report on the kinetics and vasodilator potency of authentic NO in the human forearm circulation and demonstrate that sterile aqueous NO solutions can be administered intra-arterially in humans without complications or unwanted side effects. NO in saline solution dilates conduit and resistance arteries at doses lower than those currently used in NO inhalation therapy. The observed biological effects lasted considerably longer than ex vivo studies on its half-life in blood had predicted. Intravascular application of authentic $\mathrm{NO}$ was found to lead to the in vivo formation of plasma nitrite, nitrate, and $S$-nitrosothiols, and only the latter, together with free NO, appears to be involved in the flow response elicited.
Dilator effects of authentic NO. Effects of intra-arterially applied NO on blood flow were unexpected and, to our knowledge, had not been reported before. NO appears to have a uniform effect along the vascular tree, in that it dilated conduit and resistance arteries, which are regulated in a mutually independent manner. The observation that dilation of the radial artery occurred prior to the dilator action on resistance vessels rules out that initial radial artery dilation was mediated by an endothelium-dependent, flow-mediated mechanism. Nevertheless, an additional flow-mediated component secondary to the NO-mediated increase in flow in the downstream microvasculature may contribute to the prolonged dilation of the radial artery. Alternatively, counter-regulatory vascular signaling mechanisms may reverse the NO-induced dilation in resistance arteries, which are also critically controlled by the tissue supply/demand ratio for nutrients. Conduit arteries are less sensitive to such opposing mechanisms. The duration of flow increase, and thus the biological half-life of NO, was much longer than expected from the half-life of $\mathrm{NO}$ estimated from ex vivo studies in blood $(5,6,29)$. This may be due to the longer-lasting activation of vascular downstream signaling cascades, formation of intermediate NO adducts (see below), or a reduced NO consumption under flow conditions.

In the absence of RBCs, the half-life of $\mathrm{NO}$ in aerated buffer solutions or plasma has been reported to be in the range of $1.5-6$ minutes $(5,30)$. In a static system, RBCs act as an effective sink for NO, reducing its half-
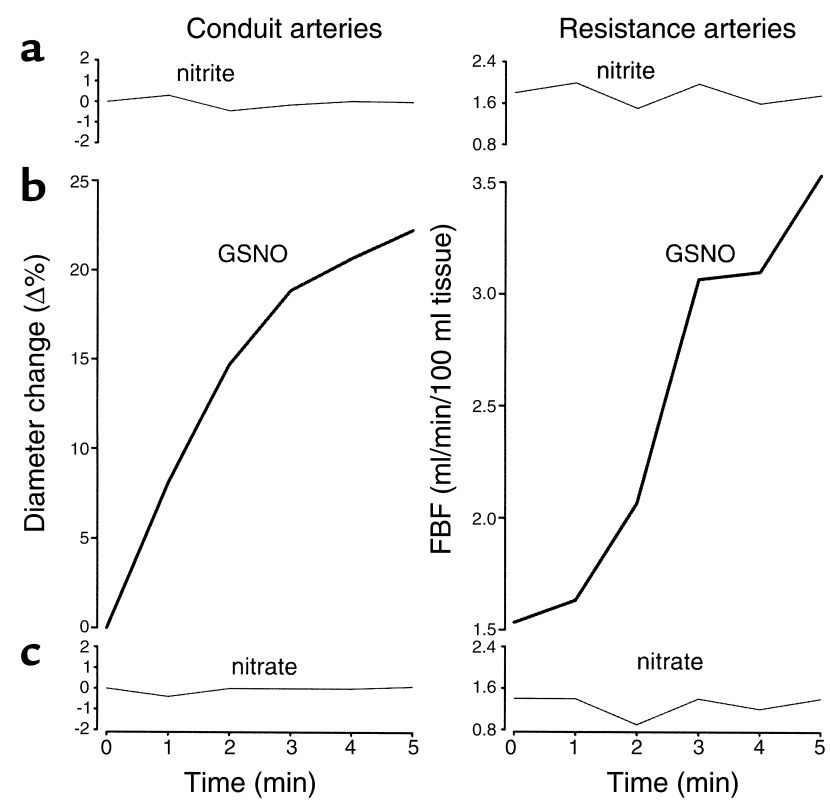

\section{Figure 5}

Dilator response of conduit and resistive arteries in the human forearm circulation following infusion of NO-related metabolites. Changes in radial artery diameter and FBF were recorded simultaneously using high-resolution ultrasound and plethysmography in three individuals during application of (a) nitrite $(36 \mu \mathrm{mol} / \mathrm{min}),(\mathbf{b})$ GSNO $(5 \mathrm{nmol} / \mathrm{min})$, or (c) nitrate $\left(\mathrm{NO}_{3}^{-} ; 36 \mu \mathrm{mol} / \mathrm{min}\right)$. 
life in plasma to something on the order of 1.8 milliseconds (5). However, the consumption of NO by $\mathrm{RBCs}$ has been suggested to be reduced due to the presence of an unstirred layer of plasma around RBCs, resulting in an effective diffusional resistance (6). Therefore, intraerythrocytic hemoglobin reacts with NO up to 1000 times more slowly than does free hemoglobin. Consumption of plasma NO by RBCs is further hampered due to flow-related effects. Using an isolated microvessel preparation, Liao et al. (31) have demonstrated that consumption of NO by RBCs progressively decreases with increasing flow, suggesting that under these conditions hemoglobin contained in RBCs is not acting as an effective NO scavenger. The effect of flow on NO consumption by RBCs may be attributed to the formation of an RBC-free zone near the vessel wall, which has been estimated to reach up to $25 \%$ of the luminal diameter in thickness (32).

Taken together, these experimental data suggest that the lifespan of $\mathrm{NO}$ in plasma in vivo is in the range of seconds to minutes and that NO may thus be transported as such at considerable distances along the vascular bed. Our data are in agreement with this interpretation, since the onset of flow increases after intra-arterial application of aqueous NO solutions occurred within seconds and was almost identical to the response kinetics following stimulation of endogenous NO synthesis in the same vascular bed (Figure 3). This suggests that at least the initial part of the vascular effects observed in the present study was mediated by NO itself.

Metabolism of NO and nitrosation chemistry in vivo. Intra-arterial NO application resulted in an increase in plasma levels of nitrite, nitrate, and RSNOs, with nitrate representing the highest and RSNOs the smallest percentage of the recovered $\mathrm{N}$-oxides. About $30 \%$ of the applied NO dose was not recovered. One possible explanation for this is that a part of the applied NO may have been converted to metabolites other than the ones measured by us or entered blood cells and tissue and thus gone undetected. Alternatively, since this portion consisted mainly of NO, it might have escaped into ambient air before becoming oxidized to nitrite or converted to nitrate, although this possibility is admittedly speculative.

In a previous study performed in the same vascular bed, we have demonstrated that plasma nitrite rather than nitrate levels reflect regional changes in eNOS activity (10). These findings suggested that NO, which is released from the endothelium into the RBCfree plasma zone lining the vascular wall, is subject to oxidative decomposition in a manner independent of hemoglobin. In line with this notion, we now demonstrate that, after application of aqueous NO solution into the human forearm vasculature, significant amounts of $\mathrm{NO}$ can be recovered as nitrite in the draining antecubital vein. However, as plasma nitrite accounted for only $5 \%$ of the amount of NO infused, alternative metabolic routes have to be considered.
The net increase in plasma nitrate levels $(>90 \mu \mathrm{mol} / \mathrm{l})$ was more than tenfold higher than that of nitrite (>8 $\mu \mathrm{mol} / \mathrm{l}$; Figure 4). Several potential routes of nitrate formation have to be considered: Conversion of nitrite to nitrate within the erythrocyte is considered unlikely to contribute much to the increase in plasma nitrate levels, because both nitrite uptake by RBCs and nitrite oxidation by hemoglobin are relatively slow processes $(33,34)$ that far exceed total passage time through the forearm circulation, which is in the range of 4-10 seconds (28). It appears rather unlikely that such a conversion took place during blood sampling or sample processing (in an unstirred blood sample), since samples were drawn within seconds, almost instantly cooled down using chilled vials, and diluted. However, if our hypothesis was correct and NO transported in plasma as such, the possibility that part of the applied NO might have entered RBCs and been converted to nitrate during sample processing cannot be excluded. Whatever the mechanism of nitrate formation, the complete lack of vasodilator activity of intra-arterially infused nitrite and nitrate rules out any role for these metabolites in NO delivery under normoxic conditions.

Earlier studies have demonstrated that the interaction of NO with albumin results in the formation of SNOAlb and that the latter represents the principal nitrosothiol in plasma $(13,14)$. We therefore made no further attempt to dissect the minor fraction of low-molecular weight RSNOs from the total plasma RSNO pool. In the present investigation, we determined basal RSNO levels to be in the low nanomolar range, which is in agreement with most other recent studies in human plasma (14). Whether nitrosation in vivo takes place exclusively at the level of plasma constituents or includes circulating blood cells as well remains to be investigated. Despite a net increase of only $30 \mathrm{nM}$ (Figure 4), the formation of RSNOs appears to account, at least in part, for the dilator effects of NO on conduit and resistance arteries. On first sight, the increases in plasma RSNOs observed during NO infusion might appear disappointingly small. However, it is important to note that comparable concentrations of GSNO $(5 \mathrm{nmol} / \mathrm{min}$ infused to a flow of $\sim 200 \mathrm{ml} / \mathrm{min}$ in the brachial artery corresponds to $25 \mathrm{nM}$ ) closely matched the vasodilator profile of authentic NO. While the onset of vasodilation seen with GSNO was delayed compared with that of the NO infusion, the sustained vasodilation observed with $\mathrm{NO}$ (Figure 3) may be mediated by the in vivo formation of plasma RSNOs. GSNO was chosen over SNOAlb for reasons of simplicity of application (no need for separation and nitrosation of autologous albumin) and to avoid possible unwanted immunological reactions due to increased amounts of protein-bound SNO epitopes. The possibility that part of the NO-induced dilation in our experiments was mediated by SNOHb (23) or NOHb (20) cannot be excluded at present, but it appears rather slight, because intraerythrocytic hemoglobin reacts with NO up to 
1000 times more slowly than does free hemoglobin, and consumption of plasma NO by RBCs is further hampered due to flow-related effects (31). However, these pathways have not been specifically addressed in our study, and further investigations are warranted to elucidate the potential role of hemoglobin in the transport of NO under physiological conditions.

Therapentic implications. Collectively, we have demonstrated that authentic NO, when applied as aqueous solution into the human artery, exerts local vasodilator effects at significant distances from the site of application. Part of these effects appears to be mediated via free NO, another part via nitrosation of plasma constituents. Systemic application of authentic NO may compensate for the NO deficiency seen in various cardiovascular diseases associated with endothelial dysfunction. The route of administration and the chosen dose may critically determine NO transport and biological effects. The latter depend on uptake, transport, and liberation of $\mathrm{NO}$ along the vascular tree by physiological blood-borne carriers, the intercellular contact of blood cells during passage through the microvasculature, and the interaction of plasma NO stores with the endothelium and vascular smooth muscle. The metabolism and distribution of $\mathrm{NO}$ and thus its range of action may exhibit significant spatial heterogeneity in vivo, depending on vessel size, hematocrit, flow velocity, and shear rate $(35,36)$ as well as oxygen saturation and tissue $\mathrm{PO}_{2}$. These variables are expected to affect $\mathrm{NO}$ consumption in the RBC-free plasma zone near the vascular wall, and within RBCs $(31,37)$. Matters are further complicated by the fact that the RBC membrane represents a heterogenous sink $(5,38)$, which may critically determine the effects of $\mathrm{NO}$ on the microcirculation (39). These denominators might be affected differently by intra-arterial, intravenous, or inhaled NO substitution therapy. The dose range required for NO-induced vasodilation in humans was hitherto unknown. The doses of NO applied in the present study are considerably lower than those in current use for inhalation therapy of critically ill patients (40). Those patients typically receive $25-35$ parts per million NO, resulting in a net uptake of 5.5-7.7 $\mu \mathrm{mol}$ NO per minute of inhalation. In a recent study aimed to assess the formation of $\mathrm{NOHb}$ and potential peripheral vasodilator effects (22), $\mathrm{NO}$ was inhaled at $80 \mathrm{ppm}$ for 1 hour, resulting in a net uptake of $1080 \mu \mathrm{mol} \mathrm{NO}$, a dose more than 30 -fold higher than the highest dose applied in the present study. Yet the magnitude of the peripheral dilator effects of NO was less than those described in the present study. Whether the application of aqueous NO solution may offer therapeutic advantages over existing NO-related therapies and furthermore be suitable to exert systemic biological effects in vivo remains to be investigated.

\section{Acknowledgments}

This work was supported by the Biomedizinisches Forschungszentrum, Heinrich-Heine Universität Düs- seldorf; by grants from the Deutsche Forschungsgemeinschaft (DFG: Pr 634/2, Ke 405/4, and SFB 612) to M. Preik and M. Kelm; and by funds from the Feist Endowment (to M. Feelisch). T. Rassaf is a research fellow funded by the DFG (RA 969/1-1). M. Kelm is a recipient of a Gerhard-Hess-Fellowship from the DFG. The technical assistance of S. Matern, C. Ferfers, and G. Doemer is gratefully acknowledged.

1. Moncada, S. 1997. Nitric oxide in the vasculature: physiology and pathophysiology. Ann. NY Acad. Sci. 811:60-69.

2. Harrison, D.G. 1997. Cellular and molecular mechanisms of endothelial cell dysfunction. J. Clin. Invest. 100:2153-2157.

3. Sessa, W.C. 1994. The nitric oxide synthase family of proteins. J. Vasc. Res. 31:131-143.

4. Borland, C. 1991. Endothelium in control. Br. Heart J. 66:405.

5. Liu, X., et al. 1998. Diffusion-limited reaction of free nitric oxide with erythrocytes. J. Biol. Chem. 273:18709-18713.

6. Vaughn, M.W., Huang, K.-T., Kuo, L., and Liao, J.C. 2000. Erythrocytes possess an intrinsic barrier to nitric oxide consumption. J. Biol. Chem. 275:2342-2348.

7. Yoshida, K., Kasama, K., Kitabatake, M., Okuda, M., and Imai, M. 1980. Metabolic fate of nitric oxide. Int. Arch. Occup. Environ. Health. 46:71-77.

8. Wennmalm, A., et al. 1993. Metabolism and excretion of nitric oxide in humans. An experimental and clinical study. Circ. Res. 73:1121-1127.

9. Kelm, M. 1999. Nitric oxide metabolism and breakdown. Biochim. Biophys. Acta. 1411:273-289.

10. Lauer, T., et al. 2001. Plasma nitrite rather than nitrate reflects regional endothelial nitric oxide synthase activity but lacks intrinsic vasodilator action. Proc. Natl. Acad. Sci. USA. 98:12814-12819.

11. Jocelyn, P.C. 1972. Biochemistry of the SH group: the occurrence, chemical properties, metabolism and biological function of thiols and disulphides. Academic Press. London, United Kingdom/New York, New York, USA. 404 pp.

12. Stamler, J.S., et al. 1992. S-nitrosylation of proteins with nitric oxide: synthesis and characterization of biologically active compounds. Proc. Natl. Acad. Sci. USA. 89:444-448.

13. Stamler, J.S., et al. 1992. Nitric oxide circulates in mammalian plasma primarily as an S-nitroso adduct of serum albumin. Proc. Natl. Acad. Sci. USA. 89:7674-7677.

14. Marley, R., et al. 2001. Formation of nanomolar concentrations of Snitroso-albumin in human plasma by nitric oxide. Free Radic. Biol. Med. 31:688-696.

15. Marley, R., Feelisch, M., Holt, S., and Moore, K. 2000. A chemiluminescense-based assay for s-nitrosoalbumin and other plasma s-nitrosothiols. Free Radic. Res. 32:1-9.

16. Jourd'heuil, D., Hallen, K., Feelisch, M., and Grisham, M.B. 2000. Dynamic state of S-nitrosothiols in human plasma and whole blood. Free Radic. Biol. Med. 28:409-417.

17. Gow, A.J., Buerk, D.G., and Ischiropoulos, H. 1997. A novel reaction mechanism for the formation of S-nitrosothiol in vivo. J. Biol. Chem. 272:2841-2845.

18. Stamler, J.S., Singel, D.J., and Loscalzo, J. 1992. Biochemistry of nitric oxide and its redox-activated forms. Science. 258:1898-1902.

19. Green, L.C., et al. 1982. Analysis of nitrate, nitrite, and $\left[{ }^{15} \mathrm{~N}\right]$ nitrate in biological fluids. Anal. Biochem. 126:131-138.

20. Gladwin, M.T., et al. 2000. Relative role of heme nitrosylation and $\beta$-cysteine 93 nitrosation in the transport and metabolism of nitric oxide by hemoglobin in the human circulation. Proc. Natl. Acad. Sci. USA. 97:9943-9948.

21. Cantilena, L.R., Jr., et al. 1992. Nitric oxide hemoglobin in patients receiving nitroglycerin as detected by electron paramagnetic resonance spectroscopy. J. Lab. Clin. Med. 120:902-907.

22. Cannon, R.O., III, et al. 2001. Effects of inhaled nitric oxide on regional blood flow are consistent with intravascular nitric oxide delivery. J. Clin. Invest. 108:279-287. DOI:10.1172/JCI200112761.

23. Stamler, J.S., et al. 1997. Blood flow regulation by S-nitrosohemoglobin in the physiological oxygen gradient. Science. 276:2034-2037.

24. Pawloski, J.R., Swaminathan, R.V., and Stamler, J.S. 1998. Cell-free and erythrocytic $S$-nitrosohemoglobin inhibits human platelet aggregation. Circulation. 97:263-267.

25. Kelm, M., Preik, M., Hafner, D., and Strauer, B.E. 1996. Evidence for a multifactorial process involved in the impaired flow response to nitric oxide in hypertensive patients with endothelial dysfunction. Hypertension. 27:346-353.

26. Preik, M., et al. 2000. Automated ultrasonic measurement of human arteries for the determination of endothelial function. Ultraschall Med. 21:195-198.

27. Di Iorio, E.E. 1981. Preparation of derivates of ferrous and ferric hemo- 
globin. In Hemoglobins. E. Antonini, C. Bernadi, and E. Chiancone, editors. Academic Press Inc. Orlando, Florida, USA. 57-72.

28. Kelm, M., Preik-Steinhoff, H., Preik, M., and Strauer, B.E. 1999. Serum nitrite sensitively reflects endothelial NO formation in human forearm vasculature: evidence for biochemical assessment of the endothelial L-arginine-NO pathway. Cardiovasc. Res. 41:765-772.

29. Lancaster,J.R., Jr. 1994. Simulation of the diffusion and reaction of endogenously produced nitric oxide. Proc. Natl. Acad. Sci. USA. 91:8137-8141.

30. Ford, P.C., Wink, D.A., and Stanbury, D.M. 1993. Autoxidation kinetics of aqueous nitric oxide. FEBS Lett. 326:1-3.

31. Liao, J.C., Hein, T.W., Vaughn, M.W., Huang, K.-T., and Kuo, L. 1999. Intravascular flow decreases erythrocyte consumption of nitric oxide. Proc. Natl. Acad. Sci. USA. 96:8757-8761.

32. Schmid-Schönbein, H., Fischer, T., Driessen, G., and Rieger, H. 1979. Microcirculation. In Quantitative cardiovascular studies: clinical research application of engineering principles. N.H.C. Hwang, D.R. Gross, and D.J. Patel, editors. University Park Press. Baltimore, Maryland, USA. 353-417.

33. Kosaka, H., Imaizumi, K., Imai, K., and Tyuma, I. 1979. Stoichiometry of the reaction of oxyhemoglobin with nitrite. Biochim. Biophys. Acta. 581:184-188.
34. May, J.M., Qu, Z.-C., Xia, L., and Cobb, C.E. 2000. Nitrite uptake and metabolism and oxidant stress in human erythrocytes. Am.J. Physiol. Cell Physiol. 279:C1946-C1954.

35. Pearson, M.J., and Lipowsky, H.H. 2000. Influence of erythrocyte aggregation on leukocyte margination in postcapillary venules of rat mesentery. Am. J. Physiol. Heart Circ. Physiol. 279:H1460-H1471.

36. Lacza, Z., et al. 2001. The cerebrocortical microcirculatory effect of nitric oxide synthase blockade is dependent upon baseline red blood cell flow in the rat. Neurosci. Lett. 291:65-68.

37. Butler, A.R., Megson, I.L., and Wright, P.G. 1998. Diffusion of nitric oxide and scavenging by blood in the vasculature. Biochim. Biophys. Acta. 1425:168-176.

38. Liu, X., Miller, M.J.S., Joshi, M.S., Thomas, D.D., and Lancaster, J.R. 1998 Accelerated reaction of nitric oxide with $\mathrm{O}_{2}$ within the hydrophobic interior of biological membranes. Proc. Natl. Acad. Sci. USA. 95:2175-2179.

39. Vaughn, M.W., Kuo, L., and Liao, J.C. 1998. Effective diffusion distance of nitric oxide in the microcirculation. Am. J. Physiol. Heart Circ. Physiol. 274:H1705-H1714.

40. Rossaint, R., et al. 1993. Inhaled nitric oxide for the adult respiratory distress syndrome. N. Engl. J. Med. 328:399-405. 